\title{
Role of fibroblast growth factor 4 in the growth and metastasis of colorectal cancer
}

\author{
YANWEI YE ${ }^{1 *}$, DONGBAO JIANG ${ }^{1 *}$, JIE LI $^{1 *}$, CHAO HAN ${ }^{2}$, \\ XINRU WANG ${ }^{2}$, FENG WANG ${ }^{3}$ and JINGJING LI ${ }^{4}$ \\ Departments of ${ }^{1}$ Gastrointestinal Surgery and Institute of Clinical Medicine, ${ }^{2}$ Pharmacy, \\ ${ }^{3}$ Pathology and ${ }^{4}$ Gastroenterology, Institute of Clinical Medicine, The First Affiliated Hospital of \\ Zhengzhou University, Zhengzhou University, Zhengzhou, Henan 450003, P.R. China
}

Received July 27, 2019; Accepted February 20, 2020

DOI: $10.3892 /$ ijo.2020.5029

\begin{abstract}
The role of fibroblast growth factor receptor 4 (FGFR4) in colorectal cancer (CRC) is poorly characterized. Therefore, the objective of the current study was to investigate the expression levels of FGFR4 in colorectal cancer and its prognostic value, and clarify the role of FGFR4 in the proliferation and metastasis of colorectal cancer cells. Immunohistochemistry was used to detect the association between FGFR4 expression and clinicopathological features in colorectal cancer tissues. The effect of FGFR4 silencing on tumor cell proliferation, cell cycle, apoptosis, migration and invasion was evaluated via lentiviral transfection of the colorectal cancer cell line SW620. Western blot analysis was used to detect the changes of epithelial-mesenchymal transition (EMT) markers, following FGFR4 silencing. FGFR4 is upregulated in CRC tissues compared with normal tissues. Patients with high FGFR4 expression exhibited a lower 5-year survival rate compared with patients with low FGFR4 expression (64 vs. 74\%). FGFR4 silencing reduced proliferation, inhibited cell invasion, arrested cells in $\mathrm{S}$ phase and promoted apoptosis in colorectal cancer cells. FGFR4 silencing partially reversed EMT progression and FGFR4 this effect was enhanced in the presence of XAV939 (a $\beta$-catenin inhibitor). The current data suggest that FGFR4 may be associated with prognosis in patients with colorectal cancer. In vitro functional tests revealed that FGFR4 may represent an effective thera-
\end{abstract}

Correspondence to: Dr Yanwei Ye, Department of Gastrointestinal Surgery and Institute of Clinical Medicine, The First Affiliated Hospital of Zhengzhou University, 1 Eastern Jian-She Road, Zhengzhou, Henan 450003, P.R. China

E-mail: yeyanwei66@126.com

*Contributed equally

Abbreviations: CRC, colorectal cancer; FGFR4, fibroblast growth factor receptor 4; EMT, epithelial-mesenchymal transition

Key words: FGFR4, colorectal cancer, proliferation, Wnt/ $\beta$-catenin, EMT peutic target for colorectal cancer. FGFR4 may also regulate EMT via the Wnt/ $\beta$-catenin pathway.

\section{Introduction}

Colorectal cancer (CRC) is one of the most common gastrointestinal malignancies, and the second most common cause of cancer-associated mortality in the United States in 2016 (1). Growth factor receptors are therapeutic targets in numerous malignancies, and dysregulation of the fibroblast growth factor receptor (FGFR) serves an important role in the genesis, progression and metastasis of colorectal cancer (2). FGFR4 (a member of the FGFR family) serves a pivotal role in the regulation of cell proliferation, differentiation, migration and angiogenesis (3-5); however, the mechanism underlying the role of FGFR4 in colorectal cancer has not yet been elucidated.

It has previously been revealed that FGFR4 is highly expressed in gastric cancer tissues, and patients with high FGFR4 expression exhibited a poor prognosis (6). BLU9931 is a FGFR4 inhibitor and significantly inhibits colorectal cancer cell proliferation, while increasing the rate of apoptosis (7). Moreover, it was reported that FGFR4 was a prognostic indicator of advanced-stage, high-grade serous ovarian cancer, and knockdown of FGFR4 using siRNA significantly inhibited the growth of ovarian tumors in vivo and in vitro (8). FGF19 is a specific agonist of FGFR4 (9). Notably, treatment of hepatoma cell lines with recombinant FGF19 protein resulted in an increase in cell proliferation and invasion; however, this tendency was partially reversed when FGFR4 was knocked down (10). This suggests that FGFR4 may represent a key target for the treatment of colorectal cancer.

Tumor recurrence and metastasis significantly impact the survival of patients, and $\sim 50 \%$ of patients with colorectal cancer eventually succumb to the disease following local recurrence and metastasis (11). Epithelial-mesenchymal transition (EMT) facilitates tumor cells to acquire invasive and metastatic potential (12). The loss of E-Cadherin protein function is a crucial step in the EMT process (13). Zhao et al (14) demonstrated that FGFR4 knockdown in hepatoma cells attenuated EMT progression by enhancing E-cadherin expression. Abnormal activation and dysregulation of Wnt/ $\beta$-catenin pathway can result in tumorigenesis. The 
abnormal accumulation of $\beta$-catenin in the nucleus is markedly associated with the occurrence of EMT (15). Knockdown of $\beta$-catenin promotes the expression of E-cadherin to reverse EMT progression in prostate cancer cells (16). However, few studies have investigated whether FGFR4 influences EMT progression via the $\mathrm{Wnt} / \beta$-catenin signaling pathway in colorectal cancer.

To explore the mechanism underlying the role of FGFR4 in tumorigenesis, progression and EMT of colorectal cancer, the expression of FGFR4 in colorectal cancer tissues and adjacent normal colorectal tissues was examined by immunohistochemistry (IHC) staining. The possible association between FGFR4 protein expression and clinicopathological features and prognosis of patients with CRC was evaluated. Furthermore, FGFR4 was silenced in the colorectal cancer cell line SW620 by constructing a small hairpin RNA (shRNA) lentivirus. A series of in vitro functional assays were performed to investigate the effect of FGFR4 silencing on colorectal cancer cells. XAV-939 selectively inhibits Wnt/ $\beta$-catenin-mediated transcription by inhibiting tankyrase1/2, and also promotes $\beta$-catenin degradation by stabilizing Axin and inhibiting Wnt/ $\beta$-catenin signaling $(17,18)$. Finally, the effect of FGFR4 on EMT progression was explored by inhibiting the Wnt/ $\beta$-catenin signaling pathway using the $\beta$-catenin inhibitor XAV-939.

\section{Materials and methods}

Tissue microarray and immunohistochemistry. Human CRC tissue microarray slides (cat. no. HColA160Su02) were purchased from Shanghai Outdo Biotech Co., Ltd. and included 100 CRC tissues and 60 adjacent normal tissues. All patients had undergone surgery between July 2005 and December 2010 and were followed up by an outpatient visit and telephone call every 6 months until September 2015. The present study was approved by the Ethics Committee of Shanghai Outdo Biotech Co., Ltd. The patients were recruited by several hospitals in Shanghai, and all provided informed written consent for research. For immunohistochemistry (IHC), FGFR4 staining was performed using a polyclonal rabbit anti-FGFR4 antibody (ProteinTech Group, Inc.; cat. no. 11098-1-AP; 1:50) as previously described (4). The stained tissue microarray was scanned using a Panoramic Midi automatic slide scanner (3DHISTECH, Hungary) Ltd. and captured byimaged using 3DHISTECH PannoramicPanoramic Viewer 1.15.2. Semiquantitative scores of cytoplasmic FGFR4 immunostaining (four-grade system) as follows: 0 , negative; 1 , weak; 2 , moderate; and 3 , strong expression. High expression was defined as when the immunostaining scores were 2 and 3 , whereas low expression was when the scores were 0 and 1 . The tissue microarray was evaluated by two double-blinded independent observers who did not know the clinical data and results.

Cell lines and cell culture. Human colorectal cancer cell line SW620 was purchased from the American Type Culture Collection. Cells were cultivated in Dulbecco's modified Eagle's medium (DMEM; Medicago, Inc.) supplemented with $10 \%$ fetal bovine serum (FBS; Gibco; Thermo Fisher Scientific, Inc.), $100 \mathrm{U} / \mathrm{ml}$ of penicillin and $0.1 \mathrm{mg} / \mathrm{ml}$ of strep- tomycin (Caisson Laboratories, Inc.) at $37^{\circ} \mathrm{C}$ in a humidified atmosphere containing $5 \% \mathrm{CO}_{2}$.

Antibodies and reagents. Rabbit monoclonal anti-FGFR4 antibody (1:1,000; cat. no. 8562), anti-Caspase3 (1:1,000; cat. no. 14220$)$, anti-active- $\beta$-catenin $(1: 1,000$ : cat. no. 19807$)$, anti-Stat3 (1:1,000; cat. no. 12640), anti-CyclinD1 $(1: 1,000$; cat. no. 55506), anti-P27 ${ }^{\mathrm{kip} 1}(1: 1,000$; cat. no. 3686) and anti- $\beta$-actin $(1: 1,000$; cat. no. 4970) antibody were all obtained from Cell Signaling Technology, Inc. Rabbit polyclonal anti-FGFR4 (1:200; cat. no. 11098-1-AP) antibody was purchased from ProteinTech Group, Inc.. Secondary horseradish peroxidase-conjugated antibodies were goat anti-mouse (1:4,000; cat. no. 12-349) and goat anti-rabbit (1:5,000; cat. no. AP132) from Sigma-Aldrich; Merck KGaA. XAV-939 (10 mM; cat. no. HY-15147) was a free sample, provided by Shanghai Haoyuan Chemical Co., Ltd. and was diluted as follows: $10 \mathrm{mM}$ XAV939 to $10 \mu \mathrm{M}$ DMSO.

Lentivirus-mediated RNA interference. The pGLV3 lentiviral packaging plasmid carrying green fluorescent protein (GFP) was purchased from Shanghai GenePharma Co., Ltd. The company designed four short hairpin RNA (shRNA; cat. nos. LV3-497, LV3-1679, LV3-1750 and LV3-2074) sequences for FGFR4 silencing, in which the sequence of shRNA (cat. no. LV3-1750) with the most pronounced effect of silencing FGFR4 was 5'-GCCGACACAAGAACATCATCA-3'. A non-silencing shRNA was used as a control and its sequence was 5'-TTCTCCGAACGTGTCACGT-3'. Colorectal cancer cells were seeded in 6-well plates at a density of 3x105 cells per well. After $24 \mathrm{~h}$, the prepared lentivirus was added to transfect colorectal cancer cells according to the manufacturer's instructions. Fluorescence microscopy was used to analyze cell transfection efficiency. After $72 \mathrm{~h}$, subsequent experiments were performed.

Protein extraction and western blotting. Whole-cell lysates were prepared by direct lysis using RIPA buffer. Protein concentrations of the samples were determined using the bicinchoninic acid protein assay (Pierce; Thermo Fisher Scientific, Inc.). Protein samples (30 $\mu \mathrm{g}$ of each protein) that were boiled for 5 min were separated on a $10 \%$ SDS-polyacrylamide gel and transferred to a PVDF membrane. The membrane was blocked with phosphate-buffered saline (PBS) containing $0.05 \%$ Tween-20 and $5 \%$ non-fat dried milk for $1 \mathrm{~h}$ at room temperature and incubated with the primary antibody at $4^{\circ} \mathrm{C}$ overnight. The immunoblots were washed 3 times with PBS containing $0.05 \%$ Tween-20 and $1 \%$ non-fat dried milk. Then PVDF membranes were incubated with secondary antibodies conjugated with horseradish peroxidase against mouse IgG or rabbit IgG for $1 \mathrm{~h}$ at room temperature. The level of a particular protein in each lysate was detected using an ECL detection system (ImageQuant LAS 3000; GE Healthcare).

Wound-healing and transwell invasion assays. For wound-healing migration assays, colorectal cancer cells were plated in 6-well plates at a density of $5 \times 10^{5}$ cells per well. The cells reached $100 \%$ confluence overnight. A single scratch wound was created by dragging a $10 \mu \mathrm{l}$ plastic pipette tip over 
A
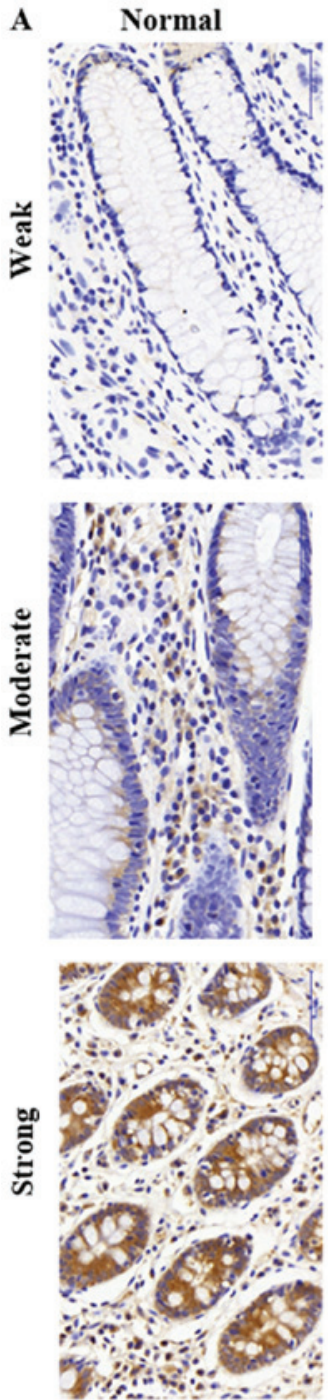

Tumor
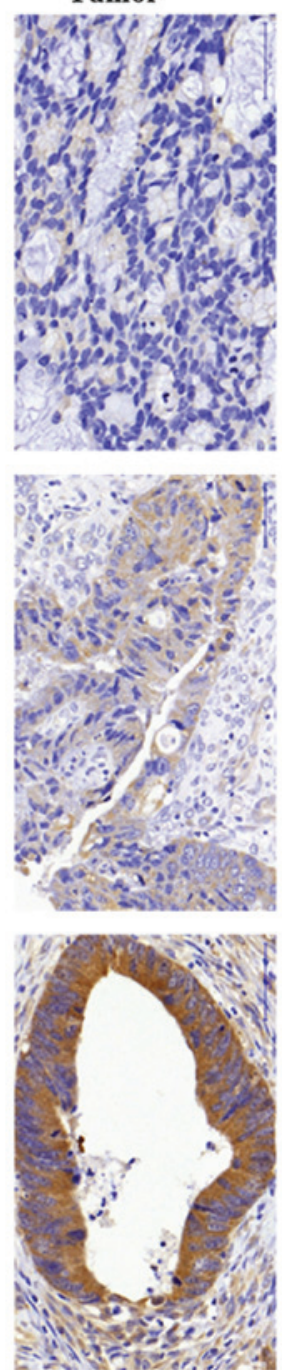

B

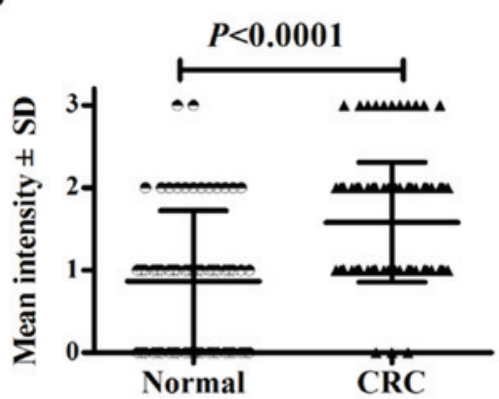

C

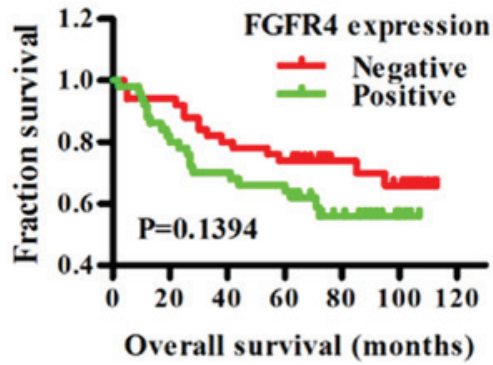

D

Invasion depth $\mathrm{T} 2+\mathrm{T} 3$

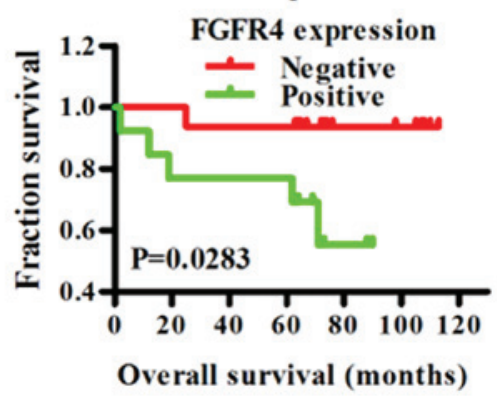

Figure 1. Expression and clinical significance of FGFR4 in colorectal cancer. (A) Immunohistochemistry for FGFR4 expression in normal colorectal and colorectal cancer tissues. Positive FGFR4 staining was primarily located in the cytoplasm. Scale bar, $100 \mu \mathrm{m}$. (B) Average staining score of FGFR4 in colorectal and CRC tissues. Expression level of FGFR4 in normal colorectal tissues was significantly compared with CRC tissues. (C) Kaplan-Meier survival curves of overall survival among patients with CRC with high or low expression of FGFR4. Subjects with FGFR4 high or low expression did not exhibit a significant difference in overall survival ( $\mathrm{P}=0.1394$ ). (D) Kaplan-Meier survival curves of the overall survival in patients with FGFR4 high or low expression at stages T2 and T3. Subgroup analyses revealed the overall survival time of patients with FGFR4 high-expression at T2 and T3 was significantly shorter compared with patients with FGFR4 low-expression $(\mathrm{P}=0.0283)$. CRC, colorectal cancer; FGFR4, fibroblast growth factor receptor 4; T, tumor.

a monolayer of cell surface. Cells were cultured in DMEM containing $2 \% \mathrm{FBS}$ at $37^{\circ} \mathrm{C}$ and $5 \% \mathrm{CO}_{2}$ for $72 \mathrm{~h}$. The degree of closure of the wound within $72 \mathrm{~h}$ was calculated and expressed as a percentage of the difference between 0 and $72 \mathrm{~h}$. The percentage of wound closure was calculated by measuring the healed area relative to the initial wound area.

For invasion assays, $8-\mu \mathrm{m}$ pore size Transwell filter inserts (Corning Inc.) covered with Matrigel (200 ng/ml; BD Biosciences) diluted by pre-cooled and serum-free DMEM medium in a 24 -well plate, and incubated at $37^{\circ} \mathrm{C}$ for $30 \mathrm{~min}$. Cells were cultured in DMEM without fetal bovine serum overnight at $37^{\circ} \mathrm{C}$. After trypsinization, cells added in the upper chamber at a density of $1 \times 10^{5}$ cells per insert. The lower chamber was filled with DMEM containing 30\% FBS. After incubation for $72 \mathrm{~h}$ at $37^{\circ} \mathrm{C}$ with $5 \% \mathrm{CO}_{2}$, the cells that invaded through a filter to the lower surface of the membrane were fixed using methanol for $10 \mathrm{~min}$ at $20^{\circ} \mathrm{C}$ and stained with
$0.2 \%$ crystal violet for $10 \mathrm{~min}$ at $20^{\circ} \mathrm{C}$. Invaded cells were counted in five randomly selected fields under a phase-contrast microscope (magnification, x200).

Proliferation assay. Cells were seeded at a density of $2 \times 10^{3}$ cells per well in the 96 -well plate (Corning Inc.) in $100 \mu \mathrm{l}$ of DMEM with $10 \%$ fetal bovine serum. After 1, 2, 3 and 4 days of culture, the cells were incubated with $5 \mathrm{mg} / \mathrm{ml}$ MTT $\left(10 \mu \mathrm{l} /\right.$ well) in a cell culture incubator at $37^{\circ} \mathrm{C}$ for $4 \mathrm{~h}$ and the supernatant was removed. DMSO (100 $\mu \mathrm{l} /$ well) was added to each well, and suspensions were agitated for $10 \mathrm{~min}$ to precipitate a purple crystal. Absorbance was measured at $490 \mathrm{~nm}$ using a microplate reader

Apoptosis detection. The rate of apoptosis in vitro was detected using Annexin V-adenomatous polyposis coli (APC)/propridium iodide (PI; Becton Dickinson and Company) 
Table I. Association between FGFR4 expression in CRC tissues and clinicopathological features.

\begin{tabular}{lccc}
\hline & \multicolumn{2}{c}{ FGFR4 expression } & \\
\cline { 2 - 3 } $\begin{array}{l}\text { Clinicopathologic } \\
\text { features }\end{array}$ & $\begin{array}{c}\text { High } \\
(\mathrm{n}=47)\end{array}$ & $\begin{array}{c}\text { Low } \\
(\mathrm{n}=48)\end{array}$ & P-value \\
\hline Sex & & & 0.7668 \\
$\quad$ Female & 26 & 28 & \\
$\quad$ Male & 21 & 20 & \\
Age, years & & & 0.3245 \\
$<60$ & 15 & 20 & \\
$\geq 60$ & 32 & 28 & \\
Lymph node metastasis & & & 0.7375 \\
N0 & 29 & 28 & \\
N1+N2 & 18 & 20 & \\
Clinical stage & & & 0.7375 \\
I+II & 29 & 28 & \\
III+IV & 18 & 20 & \\
Invasion depth & & & 0.7715 \\
T2+T3 & 17 & 16 & \\
T4 & 30 & 32 & \\
Vessel invasion & & & 0.3177 \\
No & 40 & 44 & \\
Yes & 7 & 4 & \\
\hline
\end{tabular}

FGFR4, fibroblast growth factor receptor 4; CRC, colorectal cancer.

staining. Cells were seeded at a density of $3 \times 10^{5}$ cells per well in the 6-well plate (Corning Inc.) in $2 \mathrm{ml}$ of DMEM with $10 \%$ FBS. After $24 \mathrm{~h}$ cells were harvested with EDTA-free trypsin. The cells were then incubated with Annexin V-APC and PI in the dark at room temperature for $15 \mathrm{~min}$ at $37^{\circ} \mathrm{C}$. Thereafter, all samples were analyzed by a FACSCalibur (BD Biosciences) flow cytometer with CellQuest Pro software (version 5.1; BD Biosciences). Only the AnnexinV+/PI- cells (early apoptotic) were quantified in the final graph.

Cell-cycle analysis. Cells were harvested with EDTA-free trypsin, then added the pre-cooled PBS to wash the cells twice. Cells were fixed using $70 \%$ ethanol at $4^{\circ} \mathrm{C}$ overnight. Then the cells were resuspended using $1 \mathrm{mg} / \mathrm{ml} \mathrm{PI}$ and $10 \mathrm{mg} / \mathrm{ml} \mathrm{RNase}$ A staining buffer for $15 \mathrm{~min}$ at $37^{\circ} \mathrm{C}$ in the dark. Samples were immediately analyzed using flow cytometry (ACEA NovoCyte; ACEA Biosciences, Inc.) to separate $G_{0} / G_{1}, S$, $\mathrm{G}_{2} / \mathrm{M}$ and hypodiploid nuclei. All assays were carried out in triplicate.

Statistical analysis. Statistical analysis was carried out with SPSS 13.0 (SPSS, Inc.) and GraphPad Prism 5.0 (GraphPad Software, Inc.). Staging was performed according to the American Joint Committee on Cancer (AJCC) Tumor-Node-Metastasis (TNM) staging classification for colorectal carcinoma (7th edition; 2010) (19). Results were presented as means \pm SD and three individual experiments were performed. Pearson's $\chi^{2}$ test was used to analyze the association between categorical variables. The 5-year survival rate was calculated using the Life Tables method. Survival curves were plotted according to the Kaplan-Meier method and compared using a log-rank test. Multivariate Cox regression analysis was used to determine the prognostic covariates of patients with CRC. Unpaired Student's t test was used to compare data between two groups. One-way ANOVA followed by Tukey's post-hoc test were applied to compare data between three or more groups. $\mathrm{P}<0.05$ was considered to indicate a statistically significant difference.

\section{Results}

Association between clinicopathological features and FGFR4 expression in colorectal cancer. The expression level of FGFR4 was evaluated in 100 colorectal cancer tissues and 60 adjacent normal colorectal via IHC. As exhibited in Fig. 1A, FGFR4 was expressed in colorectal cancer tissues and normal colonic tissues, and was primarily expressed in the cytoplasm. Notably, $23.3 \%$ (14/60) of normal colorectal tissue showed positive staining of FGFR4. However, 50.0\% (50/100) of CRC cases showed positive FGFR4 staining in tumor cells. The expression of FGFR4 in CRC was significantly higher compared with in normal tissues (Fig. 1B; $\mathrm{P}<0.0001$ ). Next, the association between the expression of FGFR4 and the clinicopathologic features of patients with $\mathrm{CRC}$ was evaluated. Table I reveals that the expression of FGFR4 in cancer cells was not significantly associated with sex, age, lymph node metastasis, clinical stage, invasion depth and vessel invasion.

Fig. 1C indicates the Kaplan-Meier survival curve for patients with colorectal cancer. Subjects with FGFR4 high or low expression did not exhibit significant differences in overall survival $(\mathrm{P}=0.1394)$. However, patients with FGFR4 high-expression had a lower 5-year survival time compared with patients with FGFR4 low-expression (64 vs. 74\%). Subgroup analysis revealed the overall survival time of patients with FGFR4 high-expression at stages Tumor (T)2 and T3 was significantly poorer compared with those with FGFR4 low-expression $(\mathrm{P}=0.0283)$. Univariate $\mathrm{Cox}$ regression analysis demonstrated that the overall survival rate was significantly associated with lymph node metastasis, clinical stage, invasion depth and vessel invasion (Table II; P<0.05). All statistically significant variables identified in the univariate analysis were analyzed using multivariate Cox regression. Multivariate Cox regression analysis revealed that vessel invasion was an independent risk factor for overall survival time of patients, whereas FGFR4 was not (Table II, P=0.004).

Selection of a lentivirus vector efficiently inhibiting FGFR4 expression in SW620 cells. To investigate the role of FGFR4 in the tumorigenesis of colorectal cancer lentiviral vectors were constructed containing four specific FGFR4 shRNAs (LV3-497, LV3-1750, LV3-2074 and LV3-1670) to silence the expression of FGFR4. Scrambled shRNA was used as a negative control. Fig. S1A depicts the construction of LV3 vectors. To determine the lentiviral transduction efficiency of SW620 cells, green fluorescence protein (GFP) expression was observed by fluorescence microscopy at $72 \mathrm{~h}$ after the transfec- 
Table II. Univariate and multivariate Cox regression analysis of the overall survival rate of patients with CRC.

\begin{tabular}{|c|c|c|c|c|c|c|}
\hline \multirow[b]{2}{*}{ Parameter } & \multicolumn{3}{|c|}{ Univariate } & \multicolumn{3}{|c|}{ Multivariate } \\
\hline & HR & $95 \% \mathrm{CI}$ & P-value & HR & $95 \% \mathrm{CI}$ & P-value \\
\hline \multicolumn{7}{|l|}{ Age, years } \\
\hline$\geq 60$ & 1.745 & $0.814-3.741$ & 0.152 & & & \\
\hline \multicolumn{7}{|l|}{ Sex } \\
\hline Female & 1.392 & $0.710-2.727$ & 0.335 & & & \\
\hline FGFR4 high expression & 1.678 & $0.847-3.324$ & 0.138 & 1.436 & $0.698-2.952$ & 0.138 \\
\hline \multicolumn{7}{|l|}{ Lymph node metastasis } \\
\hline $\mathrm{N} 1+\mathrm{N} 2$ & 2.351 & $1.282-4.999$ & $0.007^{\mathrm{a}}$ & 1.65 & $0.757-3.600$ & 0.208 \\
\hline \multicolumn{7}{|l|}{ Invasion depth } \\
\hline $\mathrm{T} 4$ & 2.877 & $1.190-6.954$ & $0.019^{\mathrm{a}}$ & 2.580 & 0.961-6.929 & 0.06 \\
\hline \multicolumn{7}{|l|}{ Vessel invasion } \\
\hline Yes & 3.393 & $1.699-8.470$ & $0.001^{\mathrm{a}}$ & 3.734 & $1.526-9.138$ & $0.004^{\mathrm{a}}$ \\
\hline \multicolumn{7}{|l|}{ Clinical stage } \\
\hline $\mathrm{III}+\mathrm{IV}$ & 2.351 & $1.282-4.999$ & $0.007^{\mathrm{a}}$ & 1.65 & $0.757-3.600$ & 0.208 \\
\hline
\end{tabular}

${ }^{\mathrm{a}} \mathrm{P}<0.05$. HR, hazard ratio; CI, confidence interval; FGFR4, fibroblast growth factor receptor 4; CRC, colorectal cancer.

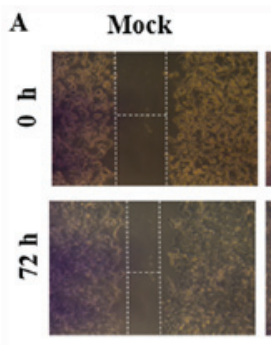

C

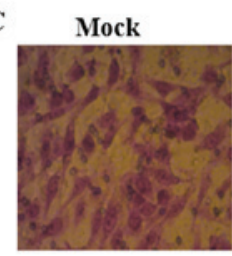

E

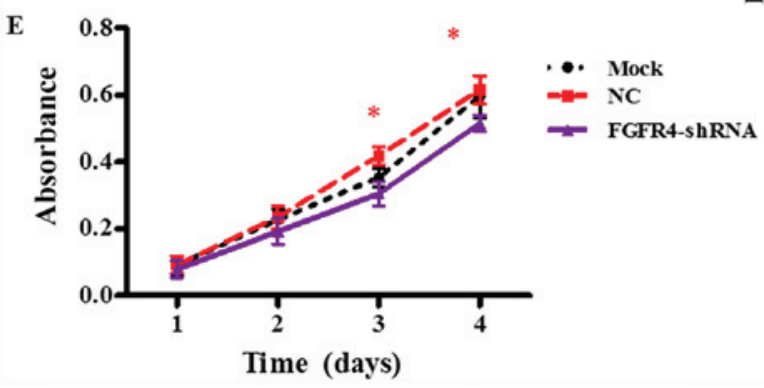

FGFR4-shRNA B
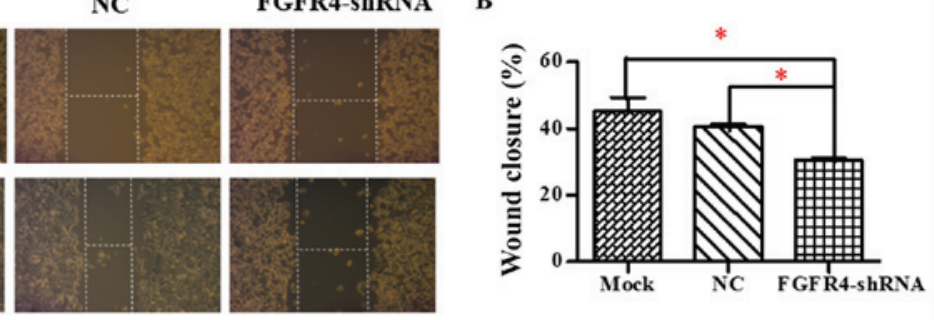

NC
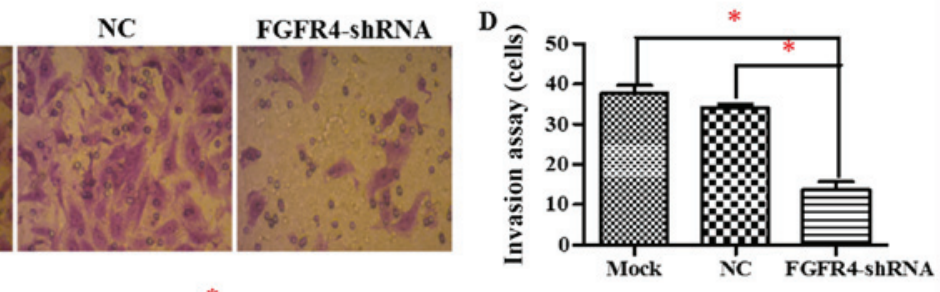

Figure 2. Effects of FGFR4 silencing on cell migration, invasion and proliferation in SW620 cells. (A) Representative images of cell density in a wound-healing migration assay. (B) Wound closure in the FGFR4-shRNA group was significantly inhibited compared with the mock and NC group. (C) Representative images of crystal violet-stained cells that migrated through the Matrigel-coated membranes. (D) Number of SW620 cells that had migrated through the Matrigel-coated membranes was significantly decreased in the FGFR4-shRNA group. (E) Cell proliferation of transfected SW620 cells was measured using MTT for 4 days. Transfection with FGFR4-shRNA significantly inhibited the proliferation ability of SW620 cells when compared with the negative and mock group, at 3 and 4 days post-transfection. All data shown are representative of 3 independent experiments. Results are expressed as mean \pm standard deviations. ${ }^{*} \mathrm{P}<0.05$. FGFR4, fibroblast growth factor receptor 4; NC, negative control; shRNA, short hairpin RNA.

tion. Lentivirus transfection in SW620 cells was demonstrated to be efficient (Fig. S1B). Western blot quantitative analysis indicated that FGFR4 protein expression in the LV3-1750 group was lowest of the four FGFR4 shRNAs, compared 

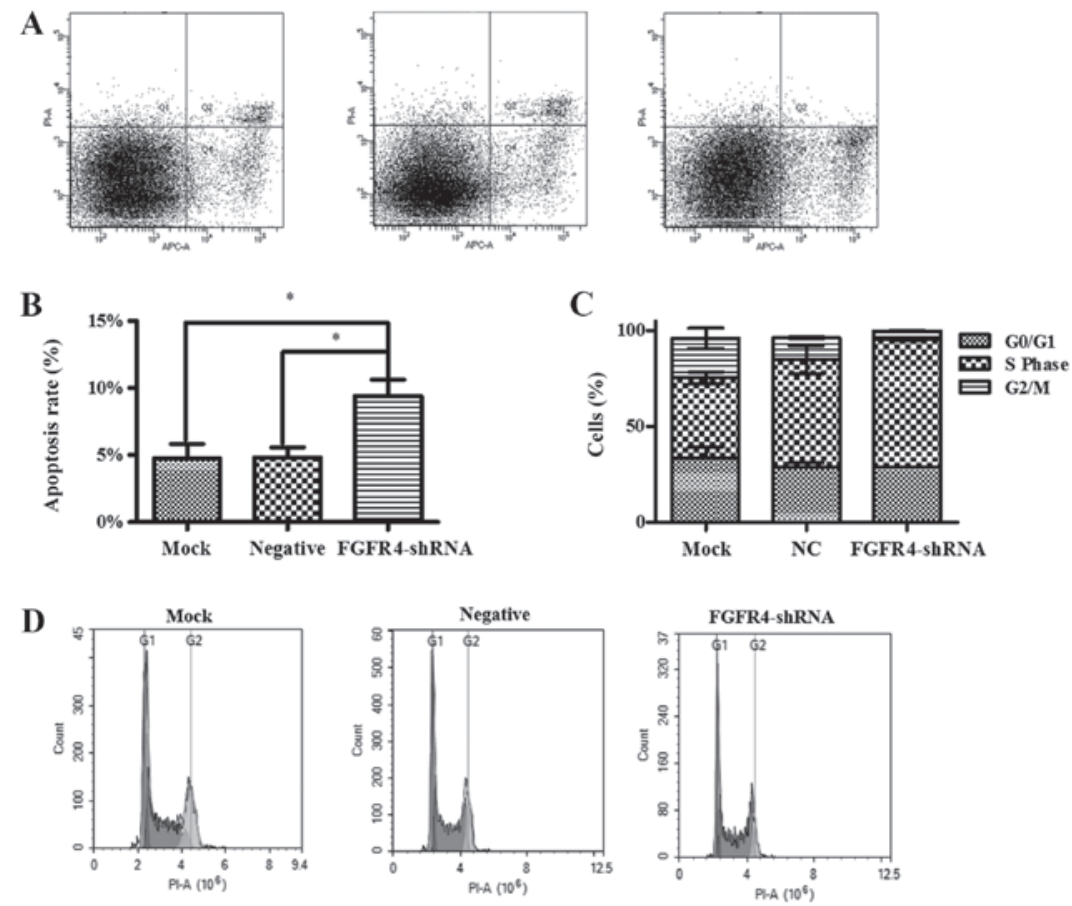

E
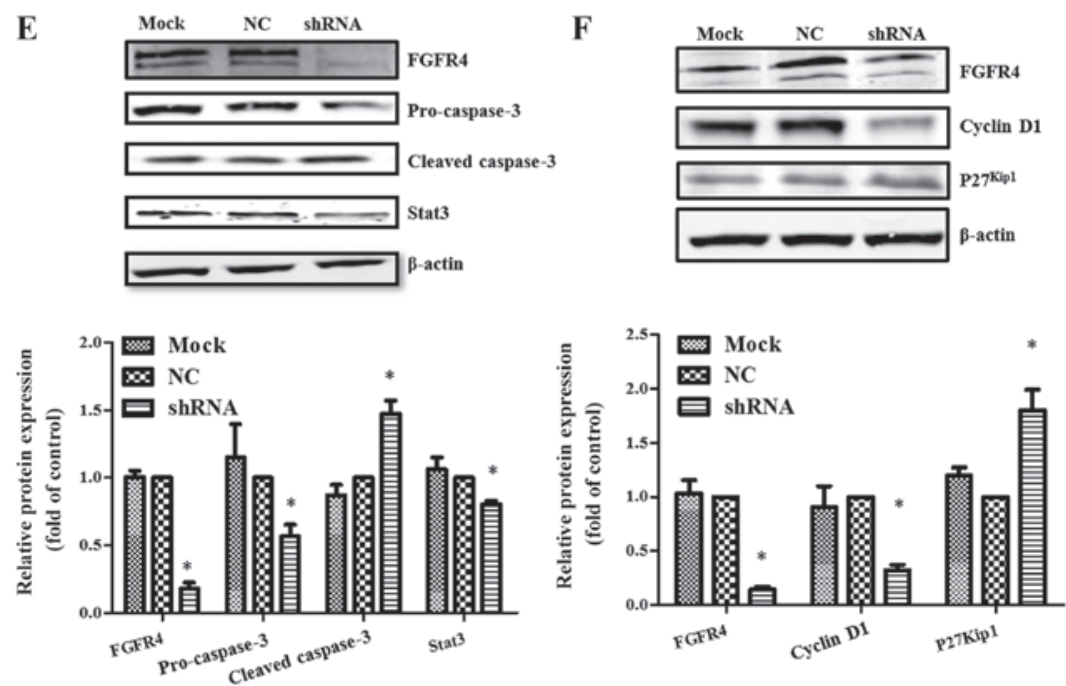

Figure 3. Effect of silencing of FGFR4 on apoptosis and cell cycle distribution in SW620 cells. (A) Flow cytometry was used to assess changes in the rate of apoptosis in SW620 cells with FGFR4 silencing. (B) Rate of apoptosis in SW620 cells that were treated with FGFR4-shRNA obviously increased compared with the negative and mock groups. (E) Western blot analysis showed that the expression of pro-caspase3, cleaved-caspase3 and stat3 in SW620 cells varied with different treatments. $\beta$-actin served as an internal control. (D) Flow cytometry was used to assess changes in the cell cycle distribution in SW620 cells with FGFR4 silencing. (C) Silencing FGFR4 in SW620 cells induced S phase cell cycle arrest after lentivirus transduction. (F) Western blot analysis demonstrated that the expression of cyclinD1 and p27 $7^{\mathrm{Kipl}}$ in SW620 cells varied with different treatments. $\beta$-actin served as an internal control. Results are expressed as means \pm standard deviations. "P<0.05 vs. NC group. FGFR4, fibroblast growth factor receptor 4; NC, negative control; shRNA, short hairpin RNA; NC, negative control; CRC, colorectal cancer.

with NC group and mock group (Fig. S1C). The above results indicated that LV3-1750 was the most effective and specific in the silencing of FGFR4, so it was selected for subsequent functional assays.

Effects of FGFR4 downregulation on migration, invasion and proliferation of SW620 Cells. The wound heal assay revealed that SW620 cells migrated into the wound scratch under a microscope (Fig. 2A). The wound closure was not significantly different between the mock group and the negative control group. However, following FGFR4-shRNA transfection, the wound closure was significantly decreased (Fig. 2B; $\mathrm{P}<0.05$ ).
This indicates that knockdown of FGFR4 is able to suppress the migration ability of SW620 cells.

The Transwell invasion assay revealed that the number of migrating cells in the mock group, negative group and the FGFR4-shRNA group were $37.67 \pm 2.08,34 \pm 1.00$ and $13.67 \pm 2.08$, respectively. In the FGFR4-shRNA group, the number of invaded SW620 cells was significantly less compared with in the mock and $\mathrm{NC}$ groups (Fig. 2C and D, $\mathrm{P}<0.05)$. However, the numbers of migrated cells in the negative group and the mock group were not significantly different.

An MTT assay was used to investigate the role of FGFR4 in cell proliferation. As demonstrated in Fig. 2E, transfection 
A
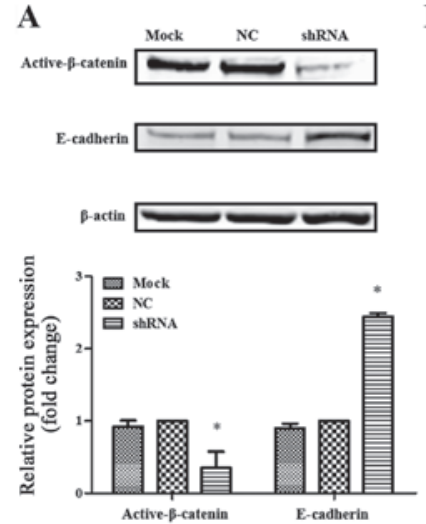

B
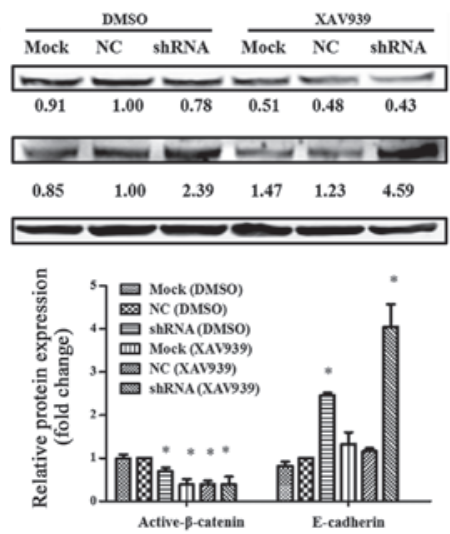

Figure 4. FGFR4 induces epithelial-mesenchymal transition via Wnt/ $\beta$-catenin signaling. (A) Western blot analysis revealed that silencing of FGFR4 in SW620 cells inhibited the activation of $\beta$-catenin and promoted the expression of E-cadherin. * $\mathrm{P}<0.05$ vs. NC group. (B) Western blot analysis indicated that the expression of E-cadherin in FGFR4-silenced SW620 cells was induced by treatment with $\beta$-catenin inhibitor XAV939 (10 $\mu$ M) for 24 h. * $<0.05$ vs. NC (DMSO-treated) group. FGFR4, fibroblast growth factor receptor 4; NC, negative control; shRNA, short hairpin RNA; CRC, colorectal cancer.

with FGFR4-shRNA significantly inhibited the proliferation ability of SW620 cells when compared with the negative and mock group $(\mathrm{P}<0.05)$, at 3 - and 4-days post-transfection.

Effects of FGFR4 downregulation on cell apoptosis and the expression of apoptotic-related proteins in SW620 cells. After $72 \mathrm{~h}$ of transfection of SW620 cells with FGFR4 shRNA, flow cytometry was used to assess the change in apoptotic rate. In SW620 cells, the apoptosis rates in the FGFR4-shRNA, NC and mock group were $9.40 \pm 1.21 \%, 4.83 \pm 0.74 \%$ and $4.77 \pm 1.05 \%$, respectively (Fig. 3A). As indicated in Fig. 3B, knockdown of FGFR4 significantly increased the apoptosis rate of SW620 cells $(\mathrm{P}<0.05)$, but there was no significant difference between the mock and NC group (Fig. 3B).

In addition, apoptosis-associated proteins Stat 3 and caspase 3 were used to assess the apoptotic capacity of SW620 cells with different treatments. In SW620 cells treated with FGFR4-shRNA, pro-caspase 3 and Stat3 expression decreased and cleaved-caspase 3 expression increased compared with both the NC group and the mock group (Fig. 3E).

Effects of FGFR4 downregulation on cell cycle distribution and cycle-related proteins in SW620 cells. After $72 \mathrm{~h}$ of transfection of SW620 cells with FGFR4 shRNA, flow cytometry was used to assess the change in cell cycle distribution (Fig. 3D). It was revealed that knockdown of FGFR4 increased the number of S-phase cells and decreased cells in the $G_{2} / M$ phase (Fig. 3C). The above results revealed that knockdown of FGFR4 blocked SW620 cells in the S phase. Subsequently, western blotting was used to detect cell cycle related proteins. The expression of $\mathrm{p} 27^{\mathrm{Kip} 1}$ in FGFR4-shRNA group was significantly increased and the expression of Cyclin D1 was significantly decreased, compared with both the NC control group and mock group.

FGFR4 influences the EMT process via the Wnt/ $\beta$-catenin signaling pathway. To explore whether the effect of FGFR4 on EMT process through the $\mathrm{Wnt} / \beta$-catenin signaling pathway, western blotting was used to detect the levels of E-cadherin and active $\beta$-catenin. As indicated in Fig. 4A, knockdown of FGFR4 in SW620 cells increased the level of E-cadherin but decreased the level of active $\beta$-catenin. Following XAV939 treatment, the levels of activated $\beta$-catenin in the mock, NC and FGFR4-shRNA groups were decreased, and were most decreased in FGFR4-shRNA group. Notably, E-cadherin levels were increased in the mock, NC and FGFR4-shRNA groups, with the highest increase in the FGFR4-shRNA group (Fig. 4B). Thus, it was hypothesized that in colorectal cancer cells, activation of FGFR4 increases the activity of $\beta$-catenin protein. Activated $\beta$-catenin protein translocates to the nucleus and initiates the expression of genes, followed by the suppression of E-Cadherin expression.

\section{Discussion}

FGFRs belong to the family of receptor tyrosine kinases (RTKs), all of which have an extracellular ligand-binding domain, a transmembrane domain and a split intracellular tyrosine kinase domain (4). The ligand-binding domain of the receptor consists of three immunoglobulin-like domains I, II and III. Immunoglobulin-like domain III is the site to which FGF binds, and domain III transcripts of FGFR1-3 undergo alternative splicing (20). Unlike FGFR1-3, FGFR4 does not alternate splicing in this region (21). By contrast, FGFR4 mutations are rare in tumor cells, but upregulation of FGFR4 is more common. To investigate the role of FGFR4 in tumorigenesis, development and metastasis, the expression level of FGFR4 in colorectal cancer tissues was examined, then FGFR4 expression was silenced in SW620 colorectal cancer cells and a series of functional assays were performed. Finally, the mechanism of action of FGFR4 in EMT was explored.

In the present study, the expression of FGFR4 in CRC was significantly higher compared with in normal tissues (Fig. 1B, $\mathrm{P}<0.0001)$. This is similartoresults reported in nasopharyngeal(22), liver (23), ovarian (8), melanoma (24) and breast cancer (25). Therefore, FGFR4 expression levels may provide valuable clues for the diagnosis of colorectal cancer. Table I reveals that the expression level of FGFR4 was not significantly associated with histopathological parameters. Combined with follow-up data, patients with FGFR4 high-expression exhibited a lower 5-year survival time than patients with FGFR4 low-expression (64 vs. 74\%), although the difference was not statistically 
significant. However, Li et al (26) reported that FGFR4 positivity was correlated with shortened disease free survival (DFS) and overall survival (OS), and that high expression of FGFR4 is an independent prognostic factor for colorectal cancer (27). The differences between the two sets of data may be caused by several factors. Primarily, in the present study, the colorectal cancer tissue sample size was relatively small compared to the aforementioned study ( $n=160$ vs. $n=316$, respectively); secondly, a tissue microarray was used for immunohistochemistry, tissue samples on the microarray experimental conditions are exactly the same, with excellent quality control; and thirdly, the present study had a longer follow-up time. Notably, subgroup analysis revealed that patients at T2 and T3 with FGFR4-positive expression had less favorable overall survival times $(\mathrm{P}=0.0283)$.

An article published previously revealed that FGFR4 protein expression is higher in HCT116 and SW620 cell lines compared with LS47,DLD1 and SW116(7). To investigate the role of FGFR4 in the tumorigenesis of colorectal cancer, lentiviral vectors were constructed containing four specific FGFR4 shRNAs (LV3-497, LV3-1750, LV3-2074 and LV3-1670) to silence the expression of FGFR4 in SW620 and HCT116. However, it was revealed that the four specific FGFR4 shRNAs were not able to silence the expression of FGFR4 in HCT116 cells (data not shown). Therefore, the present study aimed to evaluate the function of FGFR4 in the SW620. FGFR4 was silenced using shRNA and then flow cytometry was used to detect the apoptotic rate of the cells. The apoptosis rate of SW620 cells treated with shRNA was significantly increased. Western blot analysis was used to detect the expression of apoptosis-associated proteins. Consistent with the appeal, the cleaved-caspase 3 expression was significantly increased in the FGFR4-shRNA group, whereas the expression of Stat 3 and pro-caspase 3 was significantly decreased, with no significant difference between the Mock and $\mathrm{NC}$ groups. It was also demonstrated that down-regulation of FGFR4 blocked SW620 cells in S phase. Compared with the mock and NC groups, CyclingD1 as a positive regulator of the cell cycle was significantly downregulated in the FGFR-shRNA group. In the cell cycle regulatory mechanism, p27 is one of the most critical inhibitors, and inhibits the majority of CDK and cyclin complex kinase activity (27). When FGFR4 was silenced, the expression of $\mathrm{p} 27^{\mathrm{kip} 1}$ increased. MTT assays showed that silencing of FGFR4 decreased the rate of proliferation of SW620 cells. Shi et al (22) revealed that as the cell cycle progresses, the expression of FGFR4 increased; the downregulation of FGFR4 suppressed the proliferation of nasopharyngeal carcinoma cells. Therefore, it was hypothesized that FGFR4 may affect the proliferation of colorectal cancer cells by promoting apoptosis and inhibiting the cell cycle.

Tumor recurrence and metastasis adversely affect the prognosis of patients. In the current study, FGFR4 was revealed to serve an important role in the migration and invasion abilities of colorectal cancer cells. Wound scratch assays and transwell migration assays revealed that silencing FGFR4 significantly reduced the ability of SW620 cells to migrate and invade. EMT is a biological process by which epithelial cell-derived malignant cells adopt the ability to migrate and invade (12) EMT causes epithelial cells to lose the phenotype of epithelial cells and affects the expression of cell adhesion proteins such as E-Cadherin. Our previous study demonstrated that FGFR4-specific inhibitor Blu9931 induced the high expression of E-Cadherin in colorectal cancer cells (7). One study demonstrated that the FGFR4/glycogen synthase kinase $($ GSK $3 \beta) / \beta$-catenin axis may serve a key role in FGF19-induced EMT in HCC cells (14). Peláez-García et al (2) reported that FGFR4 silencing promoted the expression of E-cadherin on the surface of colorectal cancer cells via immunofluorescence analysis. FGFR4 may promote the migration and invasion of colorectal cancer cells via the EMT process.

However, the mechanism by which FGFR4 influences the EMT process is yet to be elucidated. The Wnt/ $\beta$-catenin pathway and EMT process are closely associated $(15,16)$. Disruption of the APC/Axin/GSK-3 $\beta$ complex reduces $\beta$-catenin degradation followed by activation of $\beta$-catenin into the nucleus, which is a key step in activating Wnt signaling (28). In the current study, after cells were treated with XAV939 (an inhibitor of $\beta$-catenin), E-Cadherin protein expression significantly increased compared with the no treatment FGFR4-shRNA group, while the active- $\beta$-catenin expression was significantly reduced. In colorectal cancer cells, activation of FGFR4 increases the activity of $\beta$-catenin protein. FGFR4 may influence the expression of E-Cadherin via the regulation of $\beta$-catenin. So, it is hypothesized that FGFR4 promotes EMT progression via activating the $\mathrm{Wnt} / \beta$-catenin pathway. A similar result was observed in liver cancer cells; after FGF19 agonized FGFR4, FGFR4 further activated the Wnt/ $\beta$-catenin pathway and promoted EMT progression (14).

The present study also had certain limitations. In the cell cycle regulatory mechanism, p27 is one of the most critical inhibitors. Other checkpoint proteins such as cyclin B, cyclin E, p21 and p53 should be detected. In the current study, XAV939 was used as an inhibitor of $\beta$-catenin to study the Wnt/ $\beta$-catenin pathway. The addition of agonists to the Wnt $/ \beta$-catenin signaling pathway should also be conducted to further investigate the Wnt pathway. Detection of other associated proteins influencing the Wnt/ $\beta$-catenin pathway, such as $\mathrm{N}$-Cadherin would lend support to the results of the current study. In a subsequent study, agonists of the Wnt/ $\beta$-catenin signaling pathway should be investigated, along with the expression of other checkpoint proteins of the cell cycle. To further investigate the role of FGFR4 in colorectal cancer, tumor growth analysis in nude mice should be performed.

In conclusion, in the present study it was demonstrated that FGFR4 is upregulated in human colorectal cancer tissues and appears to be associated with the prognosis of patients with colorectal cancer. It was also observed that silencing FGFR4 inhibited SW620 cell proliferation, arrested cell cycle and promoted apoptosis. In addition, the reversal of EMT progression following FGFR4 silencing may be mediated via the Wnt/ $\beta$-catenin pathway. Therefore, the results indicate that FGFR4 may be an effective target for the treatment of patients with colorectal cancer, and may also represent a useful marker.

\section{Acknowledgements}

Not applicable.

\section{Funding}

The present study was supported by the Department of Gastrointestinal Surgery and Institute of Clinical Medicine, 
The First Affiliated Hospital, Zhengzhou University (grant no. SBGJ2018010) and National Natural Science Foundation of China (grant no. 81201955).

\section{Availability of data and materials}

The datasets generated and analyzed during this study are presented in the Figures and Additional Files.

\section{Authors' contributions}

YWY and DBJ conceived and designed the study. DBJ, JL, FW, CH and XRW collected the data. YWY, DBJ, JJL and JL analyzed the data. DBJ, XRW, CH and JL performed the experiments. YWY and $\mathrm{CH}$ provided the resources and supervised the study. DBJ and JL wrote the original draft. YWY, $\mathrm{FW}, \mathrm{CH}, \mathrm{XRW}$ and JJL reviewed and edited the manuscript. All authors read and approved the final manuscript.

\section{Ethics approval and consent to participate}

The present study was approved by the ethics committee of the Shanghai Outdo Biotech Co., Ltd. (Shanghai, China). The patients were recruited by several hospitals in Shanghai, and all provided written informed consent for research. The study was undertaken in accordance with the ethical standards of the World Medical Association Declaration of Helsinki.

\section{Patient consent for publication}

Informed written consent to publish was provided by each patient that was recruited.

\section{Competing interests}

The authors declare that they have no competing interests.

\section{References}

1. Siegel RL, Miller KD and Jemal A: Cancer statistics, 2016. CA Cancer J Clin 66: 7-30, 2016.

2. Peláez-García A, Barderas R, Torres S, Hernández-Varas P, Teixidó J, Bonilla F, de Herreros AG and Casal JI: FGFR4 role in epithelial-mesenchymal transition and its therapeutic value in colorectal cancer. PLoS One 8: e63695, 2013.

3 . Turner $\mathrm{N}$ and Grose R: Fibroblast growth factor signalling: From development to cancer. Nat Rev Cancer 10: 116-129, 2010.

4. Eswarakumar VP, Lax I and Schlessinger J: Cellular signaling by fibroblast growth factor receptors. Cytokine Growth Factor Rev 16: 139-149, 2005.

5. Wang J, Stockton DW and Ittmann M: The fibroblast growth factor receptor-4 Arg388 allele is associated with prostate cancer initiation and progression. Clin Cancer Res 10: 6169-6178, 2004.

6. Ye YW, Zhou Y, Yuan L, Wang CM, Du CY, Zhou XY, Zheng BQ, Cao X, Sun MH, Fu H, et al: Fibroblast growth factor receptor 4 regulates proliferation and antiapoptosis during gastric cancer progression. Cancer 117: 5304-5313, 2011.

7. Jiang D, Li J, Li J, Wang M, Han C, Wang X, Zhao C and Ye Y: Combination of FGFR4 inhibitor Blu9931 and 5-fluorouracil effects on the biological characteristics of colorectal cancer cells. Int J Oncol 51: 1611-1620, 2017.

8. Zaid TM, Yeung TL, Thompson MS, Leung CS, Harding T, Co NN, Schmandt RS, Kwan SY, Rodriguez-Aguay C, Lopez-Berestein G, et al: Identification of FGFR4 as a potential therapeutic target for advanced-stage, high-grade serous ovarian cancer. Clin Cancer Res 19: 809-820, 2013.
9. Sawey ET, Chanrion M, Cai C, Wu G, Zhang J, Zender L, Zhao A, Busuttil RW, Yee H, Stein L, et al: Identification of a therapeutic strategy targeting amplified FGF19 in liver cancer by Oncogenomic screening. Cancer Cell 19: 347-358, 2011.

10. Miura S, Mitsuhashi N, Shimizu H, Kimura F, Yoshidome H, Otsuka M, Kato A, Shida T, Okamura D and Miyazaki M: Fibroblast growth factor 19 expression correlates with tumor progression and poorer prognosis of hepatocellular carcinoma. BMC Cancer 12: 56, 2012.

11. Punt CJ and Tol J: More is less - combining targeted therapies in metastatic colorectal cancer. Nat Rev Clin Oncol 6: 731-733, 2009.

12. Paz H, Pathak $\mathrm{N}$ and Yang J: Invading one step at a time: The role of invadopodia in tumor metastasis. Oncogene 33: 4193-4202, 2014.

13. Pez F, Lopez A, Kim M, Wands JR, Caron de Fromentel C and Merle P: Wnt signaling and hepatocarcinogenesis: Molecular targets for the development of innovative anticancer drugs. J Hepatol 59: 1107-1117, 2013.

14. Zhao H, Lv F, Liang G, Huang X, Wu G, Zhang W, Yu L, Shi L and Teng Y: FGF19 promotes epithelial-mesenchymal transition in hepatocellular carcinoma cells by modulating the GSK3 $\beta$ / $\beta$-catenin signaling cascade via FGFR4 activation. Oncotarget 7: 13575-13586, 2016.

15. Zhao JH, Luo Y, Jiang YG, He DL and Wu CT: Knockdown of $\beta$-Catenin through shRNA cause a reversal of EMT and metastatic phenotypes induced by HIF-1 $\alpha$. Cancer Invest 29: 377-382, 2011.

16. Schlessinger J: Cell signaling by receptor tyrosine kinases. Cell 103: 211-225, 2000.

17. Huang SM, Mishina YM, Liu S, Cheung A, Stegmeier F, Michaud GA, Charlat O, Wiellette E, Zhang Y, Wiessner S, et al: Tankyrase inhibition stabilizes axin and antagonizes Wnt signalling. Nature 461: 614-620, 2009.

18. Waaler J, Machon O, Tumova L, Dinh H, Korinek V, Wilson SR, Paulsen JE, Pedersen NM, Eide TJ, Machonova O, et al: A novel tankyrase inhibitor decreases canonical Wnt signaling in colon carcinoma cells and reduces tumor growth in conditional APC mutant mice. Cancer Res 72: 2822-2832, 2012.

19. Edge SB, Byrd DR, Compton CC, Fritz AG, Greene FL and Trotti A (eds): AJCC cancer staging manual. 7th edition. Springer, New York, NY, 2010.

20. Zhang X, Ibrahimi OA, Olsen SK, Umemori H, Mohammadi M and Ornitz DM: Receptor specificity of the fibroblast growth factor family. The complete mammalian FGF family. J Biol Chem 281: 15694-15700, 2006.

21. Haugsten EM, Wiedlocha A, Olsnes S and Wesche J: Roles of fibroblast growth factor receptors in carcinogenesis. Mol Cancer Res 8: 1439-1452, 2010

22. Shi S, Li X, You B, Shan Y, Cao X and You Y: High Expression of FGFR4 Enhances Tumor Growth and Metastasis in Nasopharyngeal Carcinoma. J Cancer 6: 1245-1254, 2015.

23. Ho HK, Pok S, Streit S, Ruhe JE, Hart S, Lim KS, Loo HL, Aung MO, Lim SG and Ullrich A: Fibroblast growth factor receptor 4 regulates proliferation, anti-apoptosis and alpha-fetoprotein secretion during hepatocellular carcinoma progression and represents a potential target for therapeutic intervention. J Hepatol 50: 118-127, 2009.

24. Streit S, Mestel DS, Schmidt M, Ullrich A and Berking C: FGFR4 Arg388 allele correlates with tumour thickness and FGFR4 protein expression with survival of melanoma patients. Br J Cancer 94: 1879-1886, 2006.

25. Meijer D, Sieuwerts AM, Look MP, van Agthoven T, Foekens JA and Dorssers LC: Fibroblast growth factor receptor 4 predicts failure on tamoxifen therapy in patients with recurrent breast cancer. Endocr Relat Cancer 15: 101-111, 2008

26. Li CS, Zhang SX, Liu HJ, et al: Fibroblast growth factor receptor 4 as a potential prognostic and therapeutic marker in colorectal cancer. Biomarkers 19: 81-85, 2014.

27. Conradie R, Bruggeman FJ, Ciliberto A, Csikász-Nagy A Novák B, Westerhoff HV and Snoep JL: Restriction point control of the mammalian cell cycle via the cyclin E/Cdk2:p27 complex. FEBS J 277: 357-367, 2010.

28. MacDonald BT, Tamai K and He X: Wnt/ $\beta$-catenin signaling: Components, mechanisms, and diseases. Dev Cell 17: 9-26, 2009. 